\title{
Effect of an antiviral drug control and its variable order fractional network in host COVID-19 kinetics
}

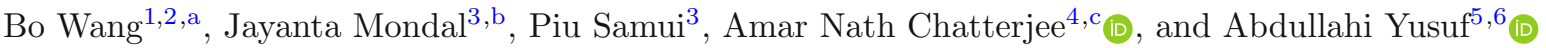 \\ 1 School of Electronic Information and Automation, Aba Teachers University, Wenchuan 623002, China \\ 2 School of Applied Mathematics, University Electronic Science and Technology of China, Chengdu 610054, China \\ 3 Department of Mathematics, Diamond Harbour Women's University, Sarisha, West Bengal 743368, India \\ 4 Department of Mathematics, K. L. S. College, Nawada, India \\ 5 Department of Computer Engineering, Biruni University, 34010 Istanbul, Turkey \\ ${ }^{6}$ Department of Mathematics, Federal University Dutse, Dutse 7156, Jigawa, Nigeria
}

Received 25 July 2021 / Accepted 13 January 2022 / Published online 1 February 2022

(C) The Author(s), under exclusive licence to EDP Sciences, Springer-Verlag GmbH Germany, part of Springer Nature 2022, corrected publication 2022

\begin{abstract}
In December 2019, a novel coronavirus disease (COVID-19) appeared in Wuhan, China. After that, it spread rapidly all over the world. Novel coronavirus belongs to the family of Coronaviridae and this new strain is called severe acute respiratory syndrome coronavirus 2 (SARS-CoV-2). Epithelial cells of our throat and lungs are the main target area of the SARS-CoV-2 virus which leads to COVID-19 disease. In this article, we propose a mathematical model for examining the effects of antiviral treatment over viral mutation to control disease transmission. We have considered here three populations namely uninfected epithelial cells, infected epithelial cells, and SARS-CoV-2 virus. To explore the model in light of the optimal control-theoretic strategy, we use Pontryagin's maximum principle. We also illustrate the existence of the optimal control and the effectiveness of the optimal control is studied here. Cost-effectiveness and efficiency analysis confirms that time-dependent antiviral controlled drug therapy can reduce the viral load and infection process at a low cost. Numerical simulations have been done to illustrate our analytical findings. In addition, a new variable-order fractional model is proposed to investigate the effect of antiviral treatment over viral mutation to control disease transmission. Considering the superiority of fractional order calculus in the modeling of systems and processes, the proposed variable-order fractional model can provide more accurate insight for the modeling of the disease. Then through the genetic algorithm, optimal treatment is presented, and its numerical simulations are illustrated.
\end{abstract}

\section{Abbreviations}

SARS-CoV-2: Severe Acute Respiratory Syndrome Coronavirus 2

WHO: World Health Organisation

ACE2: $\quad$ Angiotensin-converting enzyme 2

AIR: $\quad$ Adaptive immune responses

ICER: Incremental cost-effectiveness ratio

PRCC: Partial rank correlation coefficient

\section{Introduction}

COVID-19 is the disease that affects the whole world and this disease is caused by severe acute respiratory syndrome coronavirus 2 (SARS-CoV-2) [1]. Coronavirus can be spread and infects humans through

\footnotetext{
${ }^{a}$ e-mail: coolbie@163.com

b e-mail: jayantajumath@gmail.com (corresponding author)

c e-mail: anchaterji@gmail.com (corresponding author)
}

droplets via coughing and sneezing from infected humans. A total of 13 million people worldwide living with COVID-19 today and more than half a million people died to date. Scientists and researchers of the whole world are searching for the anti-COVID-19 vaccine. But to date, no such fruitful results have shown yet. In view of that, the use of available antiviral drugs and immunosuppressive drugs combination is the only way to fight against COVID-19 infection [2]. Therefore nonpharmaceutical interventions like self-isolation, using masks, hand washing, sanitation are suggested by the World Health Organisation (WHO) and Indian Council for Medical Research (ICMR). Also, the Government of states has implemented lockdown, travel restrictions, quarantine measures and testing to control the disease. Several epidemiological mathematical models [319] have been developed to make the right decisions in these measures. These have highlighted that social distancing intervention to mitigate the epidemic is a key aspect.

To study the COVID-19 dynamics within human host, a few mathematical models have been developed 
[2,20-24]. The major target area of the SARS-CoV-2 virus are the epithelial cells in the upper respiratory tract and upper divisions of bronchi $[2,25]$. If the infection spreads to the lower lungs, the patients' conditions become severe $[2,26,27]$. The cell infection process by the SARS $\mathrm{CoV}-2$ virus has mainly two stages. At first, the SARS-CoV-2 virus binds to the angiotensinconverting enzyme 2 (ACE2) receptors on the surface cells [28] and viral genome penetrates the cell and cells become infected. The infected cells turned into virusproducing cells and the new virions are reproduced through the eclipse and burst phase [2]. Zhou et al. [1] identified the principal infection site of the human host in COVID-19 and also reported that SARS-CoV2 spikes $\mathrm{S}$ binds with ACE2 receptor of epithelial cells with high affinity. Their study shows that the appropriate dosing interval and drug dosage are important to eradicate the viral load. Chatterjee and Bashir [23] formulated a mathematical model to examine the consequence of adaptive immune response to the viral mutation to control disease transmission. They also study the effect of the combination of antiviral drug therapy and its effect on the model dynamics. Chatterjee et al. [24] proposed a group of fractional equations model considering uninfected epithelial cells, infected epithelial cells, SARS-CoV-2 virus, and CTL response inducing cells accounting both the lytic and non-lytic effects of immune response and the effects of commonly used antiviral drugs.

In this research article, we propose mathematical modeling to investigate the SARS-CoV-2 replication within host, specifically the interaction between susceptible and SARS-CoV-2 virus. We also study the current pharmaceutical interventions in our model. Here we study the effect of commonly used antiviral and immune-suppressing drugs. It has been observed that antiviral drugs and immunosuppressive drugs play a pivotal role in the treatment of high - risk patients $[2,21]$. Immunosuppressive drugs decrease the adaptive immune responses (AIR) to its low level not to inhibit the innate immune response during the primary phase of infection $[2,21]$. Due to the effect of the immunosuppressive drugs, the infected cells become inadequate to produce new virions. The drug should be removed so that the AIRs can kill the residual virus. Insight looks at the drug regimen leads to fast and thorough recovery. Here we have formulated the basic mathematical model of COVID-19 based on drug effectiveness.

The article is designed as follows: first we have provided the formulation of our mathematical model centered with drug effectiveness in the Sect. 2 and studied the model analytically. In Sect. 3, We have framed the optimal control problem in the light of cost function for optimal drug dosage. In Sect. 4, the numerical studies of both the models (time independent and time dependent control) are depicted. Also the drug efficiency along with cost effectiveness have been verified. In Sect. 5, we proposed a variable order fractional model describing the SARS-CoV-2 infection and the effect of optimal strategies in this system. In Sect. 6, we have drawn discussions regarding our analytical and numer-

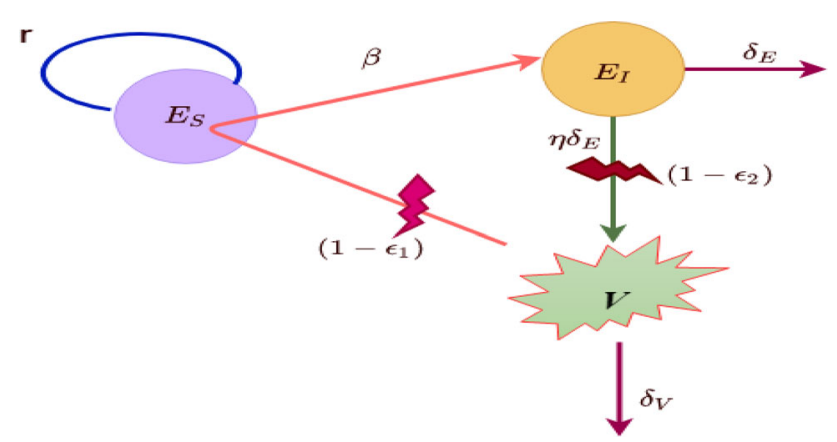

Fig. 1 The schematic figure symbolises the model of virus host infection process under combination of drug regimen

ical findings and finally in Sect. 7, we conclude about our gained results.

\section{Analysis of basic model with fixed control}

For COVID-19 infection, SARS-CoV-2 virus infects epithelial cells that carry the ACE2 receptor protein. After the cells become infected, such cells can produce new SARS-CoV-2 virus particles. To model the infection process we consider uninfected cells $\left(E_{\mathrm{S}}\right)$, infected virus-producing cells $\left(E_{\mathrm{I}}\right)$, and free virus $(V)$. The host viral infection process is yet to be explored completely. We have considered a reasonable dynamics of uninfected cells as

$$
\frac{\mathrm{d} E_{\mathrm{S}}}{\mathrm{d} t}=r E_{\mathrm{S}}\left[1-\frac{E_{\mathrm{S}}}{K}\right]
$$

where we consider that epithelial cells can be produced by proliferation from existing cells. Here we present the proliferation by a logistic function in which $r$ is the maximum proliferation rate and $K$ is the density of the epithelial cells at which proliferation shuts off.

In presence of virus, uninfected epithelial cells become infected. The simplest and common way to model infection is to augment with mass action term, $\beta E_{\mathrm{S}} V$. Infection also occurs by cell transmission when infected cells directly interact with uninfected cells. Since there is little evidence of cell infection pathway in vivo, thus we ignore this mode of infection rate here. The death rate of infected cells is $\delta_{\mathrm{E}} E_{\mathrm{I}}$ and $\eta$ is the number of virus produced from infected cells. We also consider $\delta_{V} V$ as the viral removal rate. In terms of biological view, we have considered an ordinary differential equation model that describes the interaction between epithelial cells and SARS-CoV-2. We have explored the optimal control strategy of this ODE model. Our proposed model is as follows:

$$
\begin{aligned}
\frac{\mathrm{d} E_{\mathrm{S}}}{\mathrm{d} t} & =r E_{\mathrm{S}}\left[1-\frac{E_{\mathrm{S}}}{K}\right]-\left[1-\varepsilon_{1}\right] \beta E_{\mathrm{S}} V, \\
\frac{\mathrm{d} E_{\mathrm{I}}}{\mathrm{d} t} & =\left[1-\varepsilon_{1}\right] \beta E_{\mathrm{S}} V-\delta_{\mathrm{E}} E_{\mathrm{I}}, \\
\frac{d V}{\mathrm{~d} t} & =\left[1-\varepsilon_{2}\right] \eta \delta_{\mathrm{E}} E_{\mathrm{I}}-\delta_{V} V,
\end{aligned}
$$


with this non-negative initial condition:

$$
E_{\mathrm{S}}\left(t_{0}\right)=E_{S}^{0}, \quad E_{\mathrm{I}}\left(t_{0}\right)=E_{I}^{0}, \quad V\left(t_{0}\right)=V^{0} .
$$

The model considers that $t_{0} \in[0, \infty)$ is the starting day of COVID-19 infection. Here $\varepsilon_{1}$ and $\varepsilon_{2}$ are the effectiveness of the antiviral drug with $0<\varepsilon_{1}<1,0<$ $\varepsilon_{2}<1$. $\varepsilon_{1,2}=0$ represents no antiviral drug effect while $\varepsilon_{1,2}=1$ represents that the drug is $100 \%$ effective.

\subsection{Positivity and boundedness}

Theorem 1 All the solutions of the system (2.2) subject to initial condition (2.3) are positive and bounded in $\mathbb{R}_{+}^{3}$, for all $t>0$.

Proof The first equation of the system (2.2) can be written as;

$$
\begin{aligned}
\frac{\mathrm{d} E_{\mathrm{S}}}{\mathrm{d} t} & =r E_{\mathrm{S}}\left[1-\frac{E_{\mathrm{S}}}{K}\right]-\left[1-\varepsilon_{1}\right] \beta E_{\mathrm{S}} V, \\
& =a E_{\mathrm{S}}-b E_{\mathrm{S}}^{2},
\end{aligned}
$$

where $a=\left[r-\left(1-\varepsilon_{1}\right) \beta V\right]$ and $b=r / K$. Then on integration we get,

$$
E_{\mathrm{S}}=\frac{e^{\int_{0}^{t} \mathrm{ads}}}{\int_{0}^{t} b e^{\int_{0}^{t} \mathrm{ads}} \mathrm{d} p+C} .
$$

In the similar way, it can be observed that

$$
\begin{aligned}
E_{\mathrm{I}}= & E_{\mathrm{I}}^{0} \exp \left(-\int_{0}^{t} \delta_{I} \mathrm{~d} s\right)>0 \\
& \text { and } V=V^{0} \exp \left(-\int_{0}^{t} \delta_{V} \mathrm{~d} s\right)>0 .
\end{aligned}
$$

Consequently, all the solution trajectories $\left(E_{\mathrm{S}}, E_{\mathrm{I}}, V\right)$ of system (2.2) with initial conditions (2.3) are positive. Next we show the boundedness of the solutions. According to [29], we can construct from the first and second equations of system (2.2)

$$
\limsup _{t \rightarrow \infty}\left(E_{\mathrm{S}}+E_{\mathrm{I}}\right) \leq \frac{r K}{4 \delta_{\mathrm{E}}}=\mu .
$$

Accordingly, $E_{\mathrm{S}} \leq \mu$ and $E_{\mathrm{I}} \leq \mu$. Similarly, from the third equation of the system (2.2) we can write

$$
\limsup _{t \rightarrow \infty}(V) \leq \frac{\left(1-\varepsilon_{2}\right) \eta \delta_{\mathrm{E}} \mu}{\delta_{V}}=\omega .
$$

Therefore, we get the positively invariant bounded region

$$
\Phi=\left\{\left(E_{\mathrm{S}}, E_{\mathrm{I}}, V\right) \in \mathbb{R}_{+}^{3}: E_{\mathrm{S}}, E_{\mathrm{I}} \leq \mu, V \leq \omega\right\} .
$$

The region $\Phi$ is well posed and attracting since all the solutions of the system (2.2) with initial conditions (2.3) will enter this region and will never leave it.

\subsection{Equilibria}

Here we determine the biologically feasible equilibrium points.

1. The model (2.2) have two disease-free equilibrium points, one is $P^{0}(0,0,0)$ and second one is $P_{0}(K, 0,0)$. Here we consider only disease-free equilibrium $P_{0}(K, 0,0)$ because this is very relevant for our system (2.2) with initial condition (2.3) to be biologically feasible. Disease-free equilibrium $P_{0}(K, 0,0)$ always exists.

2. $P^{*}\left(E_{\mathrm{S}}^{*}, E_{\mathrm{I}}^{*}, V\right)$ is the endemic equilibrium where COVID-19 infection persists in the system.

\subsection{Basic reproduction number}

In this subsection, we now determine the basic reproduction number $\left(R_{0}\right)$ of the system (2.2) using the next generation matrix method proposed in [30,31]. It is notable that the infected compartments of the system (2.2) are $E_{\mathrm{I}}$ and $V$. Let $\mathcal{M}$ depicts the emergence of new infections while $\mathcal{N}$ stands for transfer of the infections between the compartments and $x=\left[E_{\mathrm{I}}, V\right]^{T}$. Then at the disease-free steady state $P_{0}(K, 0,0)$ the linearized infectious subsystem of the system (2.2) is given by $\dot{x}=M-N$ where

$$
\begin{aligned}
\mathcal{M} & =\left[\begin{array}{l}
\beta_{s}\left(1-\varepsilon_{1}\right) E_{\mathrm{S}} V \\
0
\end{array}\right], \\
\text { and } \quad \mathcal{N} & =\left[\begin{array}{l}
\delta_{\mathrm{E}} E_{\mathrm{I}} \\
-\left(1-\varepsilon_{2}\right) \eta \delta_{\mathrm{E}} E_{\mathrm{I}}+\delta_{V} V
\end{array}\right] .
\end{aligned}
$$

Accordingly we can calculate that

$$
\begin{aligned}
& M=\left[\begin{array}{ll}
0 & \beta_{s}\left(1-\varepsilon_{1}\right) E_{\mathrm{S}} \\
0 & 0
\end{array}\right], \quad \text { and } \\
& N=\left[\begin{array}{cc}
\delta_{\mathrm{E}} & 0 \\
-\left(1-\varepsilon_{2}\right) \eta \delta_{\mathrm{E}} & \delta_{V}
\end{array}\right] .
\end{aligned}
$$

Therefore next generation matrix is $M N^{-1}$ and

$$
M N^{-1}=\frac{1}{\delta_{\mathrm{E}} \delta_{V}}\left[\begin{array}{cc}
\left(1-\varepsilon_{1}\right)\left(1-\varepsilon_{2}\right) \beta \eta \delta_{\mathrm{E}} K & \left(1-\varepsilon_{)} \beta \delta_{\mathrm{E}} K\right. \\
0 & 0
\end{array}\right] .
$$

And we have

$$
R_{0}=s p\left(M N^{-1}\right)=\frac{\left(1-\varepsilon_{1}\right)\left(1-\varepsilon_{2}\right) \beta \eta K}{\delta_{V}} .
$$

\subsection{Existence of the endemic steady state}

To acquire feasible solutions of the system (2.2), the equilibria of the system must be positive. The positive components of the unique endemic equilibrium $P^{*}\left(E_{\mathrm{S}}^{*}, E_{\mathrm{I}}^{*}, V^{*}\right)$ are computed as 
Table 1 Variables and parameter values used for numerical simulations of the system (2.2)

\begin{tabular}{|c|c|c|}
\hline & Variables and parameters & \\
\hline Dependent & Biological meaning & \\
\hline \multicolumn{3}{|l|}{ Variables } \\
\hline$E_{\mathrm{S}}$ & Susceptible cells & \\
\hline$E_{\mathrm{I}}$ & Infected cells & \\
\hline$V$ & Free virus & \\
\hline Parameters & Biological meaning & Assigned value (unit) \\
\hline$r$ & Growth rate of cells & $13.3 \times 10^{-1}\left(\right.$ cell $\left.\mathrm{h}^{-1}\right)$ \\
\hline$K$ & Carrying capacity of cell & $1.8 \times 10^{5}\left(\right.$ cells ml $\left.{ }^{-1}\right)$ \\
\hline$\beta$ & Rate of infection/transmission & $2.1 \times 10^{-8}(\mathrm{CFU} / \mathrm{ml})^{-1} \mathrm{~h}^{-1}$ \\
\hline$\varepsilon_{1}$ & Efficacy of immunosuppressive drug & {$[0,1]$} \\
\hline$\varepsilon_{2}$ & Efficacy of antiviral drug & {$[0,1]$} \\
\hline$\delta_{\mathrm{E}}$ & Death rate of epithelial cell & $16.7 \times 10^{-2}\left(\mathrm{~h}^{-1}\right)$ \\
\hline \multirow[t]{2}{*}{$\eta$} & Number of free virus produced & \\
\hline & from infected cells & $11.3 \times 10^{-1}$ \\
\hline$\delta_{V}$ & Virus removal rate & $0.4\left(\mathrm{~h}^{-1}\right)$ \\
\hline
\end{tabular}

Fig. 2 The system trajectories without control $\left(\varepsilon_{1}=0, \varepsilon_{2}=0\right)$ and with control (see inset figure where $\varepsilon_{1}=0.75 \neq 0, \varepsilon_{2}=$ $0.75 \neq 0$ )

Fig. 3 The forward bifurcation curves for $(2.2)$ in the $\left(R_{0}, E_{\mathrm{I}}\right)$ planes. The parameter $\beta$ varied in the range

$\left[2.1 \times 10^{-8}, 2.1 \times 10^{-6}\right]$ to allow $R_{0}$ to vary in the range $[0,5]$
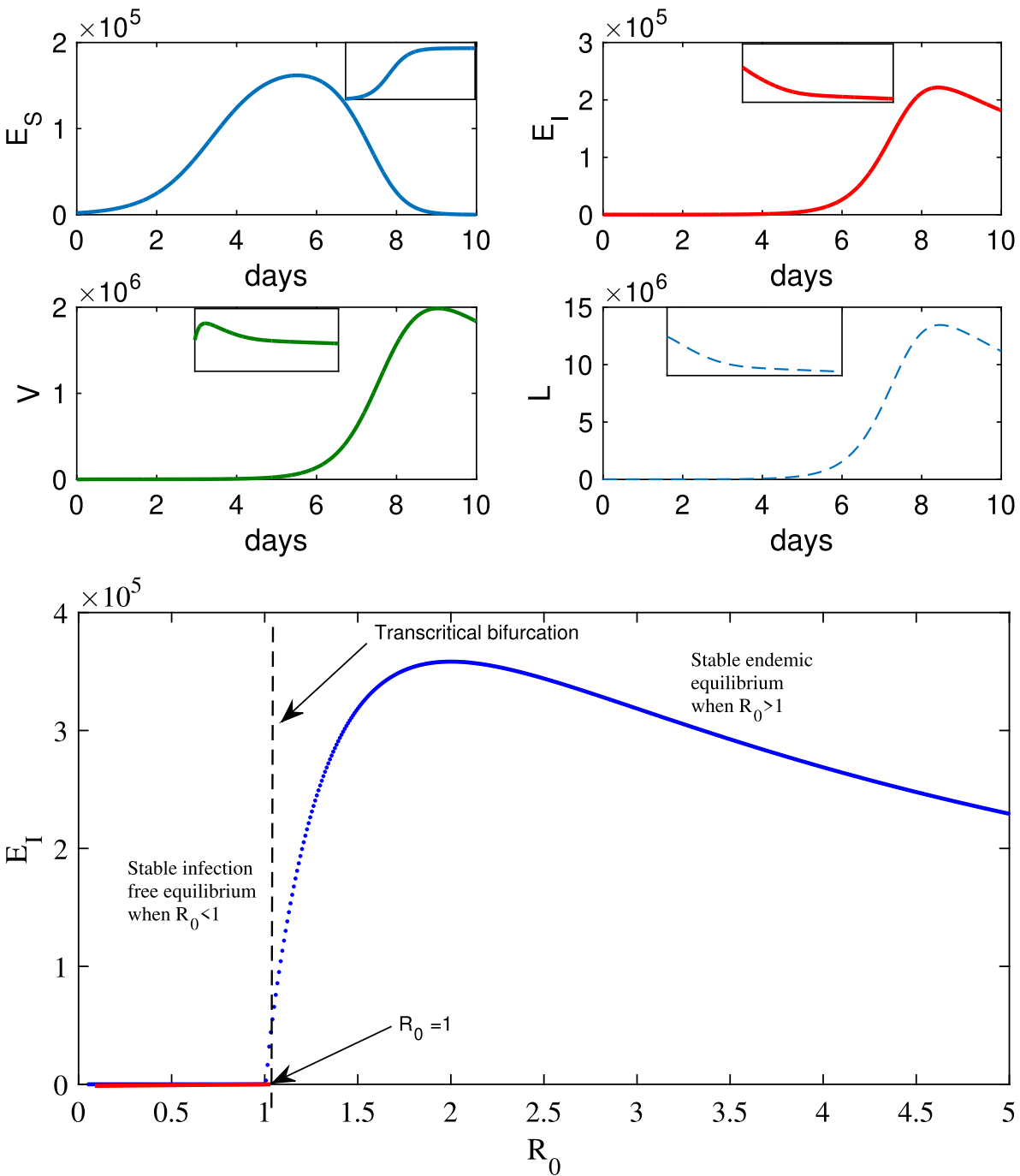


$$
\begin{aligned}
E_{\mathrm{S}}^{*} & =\frac{\delta_{V}}{\left(1-\varepsilon_{1}\right)\left(1-\varepsilon_{2}\right) \beta \eta}, \\
E_{\mathrm{I}}^{*} & =\frac{r \delta_{V}}{\left(1-\varepsilon_{1}\right)\left(1-\varepsilon_{2}\right) \beta \eta \delta_{\mathrm{E}}}\left(1-\frac{1}{R_{0}}\right), \\
V^{*} & =\frac{r \delta_{V}}{\left(1-\varepsilon_{1}\right) \beta}\left(1-\frac{1}{R_{0}}\right) .
\end{aligned}
$$

Therefore it is observed that the unique endemic equilibrium $P^{*}\left(E_{\mathrm{S}}^{*}, E_{\mathrm{I}}^{*}, V^{*}\right)$ would exist only if $R_{0}>1$.

\subsection{Local stability analysis}

Theorem 2 The system attains its locally asymptotically steady state at disease-free equilibrium point of the system (2.2) if $R_{0}<1$ and would be unstable when $R_{0}>1$.

Proof The Jacobian matrix calculated at the diseasefree equilibrium point $P_{0}(K, 0,0)$ is

$$
J_{P_{0}}=\left[\begin{array}{ccc}
-r & 0 & -\left(1-\varepsilon_{1}\right) \beta K \\
0 & -\delta_{\mathrm{E}} & \left(1-\varepsilon_{1}\right) \beta K \\
0 & -\left(1-\varepsilon_{2}\right) \eta \delta_{\mathrm{E}} & -\delta_{V}
\end{array}\right]
$$

The characteristic equation corresponding of the Jacobian matrix $J_{P_{0}}$ corresponding to the eigenvalue $\rho$ is given by

$$
\begin{aligned}
& (-r-\rho)\left[\rho^{2}+\left(\delta_{\mathrm{E}}+\delta_{V}\right) \rho+\right. \\
& \left.\quad\left\{\delta_{\mathrm{E}} \delta_{V}-\left(1-\varepsilon_{1}\right)\left(1-\varepsilon_{2}\right) \beta \delta_{\mathrm{E}} \eta K\right\}\right]=0 .
\end{aligned}
$$

Therefore we get $\rho=-r$ and the real parts of the other two eigenvalues must be negative for the locally asymptotically stable of the equilibrium $P_{0}$ and this will be possible only when $\left(\delta_{\mathrm{E}} \delta_{V}-\left(1-\varepsilon_{1}\right)\left(1-\varepsilon_{2}\right) \beta \delta_{\mathrm{E}} \eta K\right)>$ 0 i.e. $R_{0}<1$. If one of the eigenvalues or its real part would be positive, then $R_{0}>1$ and consequently $P_{0}$ would be unstable.

Theorem 3 The endemic equilibrium point $P^{*}\left(E_{\mathrm{S}}^{*}, E_{\mathrm{I}}^{*}\right.$, $\left.V^{*}\right)$ is locally asymptotically stable if $R_{0}>1$.

Proof The Jacobian matrix around the equilibrium point $P^{*}\left(E_{\mathrm{S}}^{*}, E_{\mathrm{I}}^{*}, V^{*}\right)$ is

$$
J_{P^{*}}=\left[\begin{array}{ccc}
-\frac{r E_{\mathrm{S}}^{*}}{K} & 0 & -\left(1-\varepsilon_{1}\right) \beta E_{\mathrm{S}}^{*} \\
\left(1-\varepsilon_{1}\right) \beta V^{*} & -\delta_{\mathrm{E}} & \left(1-\varepsilon_{1}\right) \beta E_{\mathrm{S}}^{*} \\
0 & \left(1-\varepsilon_{2}\right) \eta \delta_{\mathrm{E}} & -\delta_{V}
\end{array}\right]
$$

The characteristic equation of the Jacobian matrix $J_{P^{*}}$ corresponding to the eigen value $\gamma$ is given by

$$
\gamma^{3}+\varrho_{1} \gamma^{2}+\varrho_{2} \gamma+\varrho_{3}=0
$$

where,

$$
\begin{aligned}
& \varrho_{1}=\frac{r}{R_{0}}+\delta_{\mathrm{E}}+\delta_{V}, \quad \varrho_{2}=\frac{r}{R_{0}}\left(\delta_{\mathrm{E}}+\delta_{V}\right), \\
& \varrho_{3}=r \delta_{\mathrm{E}} \delta_{V}\left(1-\frac{1}{R_{0}}\right) .
\end{aligned}
$$

It is notable that $\varrho_{3}>0, \varrho_{2}>0, \varrho_{1}>0$ and $\varrho_{1} \varrho_{2}>$ $\varrho_{3}$ only for $R_{0}>1$, that is the Routh-Hurwitz criterion is satisfied for the cubic equation (2.7) and this ensures that all the three roots of the characteristic equation (2.7) have negative real parts when $R_{0}>1$. Therefore the endemic equilibrium point $P^{*}\left(E_{\mathrm{S}}^{*}, E_{\mathrm{I}}^{*}, V^{*}\right)$ is locally asymptotically stable provided $R_{0}>1$.

\subsection{Global stability analysis of disease-free equilibrium}

Theorem 4 The disease-free equilibrium point $P_{0}(K$, $0,0)$ is a globally asymptotically steady state point whenever $R_{0}<1$ and would be unstable if $R_{0}>1$.

Proof Let us define a Lyapunov function $L: \Phi \rightarrow \mathbb{R}$ for the system (2.2) as

$$
L=\frac{\left(1-\varepsilon_{2}\right) \eta}{\delta_{V}} E_{\mathrm{I}}+\frac{1}{\delta_{V}} V
$$

The Lyapunov function $L$ is positive in the non-negative cone $\Phi$ and attains zero at $P_{0}(K, 0,0)$. Taking orbital derivative of $L$ along positive solutions of the system $(2.2)$, we get

$$
\begin{aligned}
\frac{\mathrm{d} L}{\mathrm{~d} t} & =\frac{\left(1-\varepsilon_{2}\right) \eta}{\delta_{V}} \frac{\mathrm{d} E_{\mathrm{I}}}{\mathrm{d} t}+\frac{1}{\delta_{V}} \frac{d V}{\mathrm{~d} t} \\
& =\left(\frac{\left(1-\varepsilon_{1}\right)\left(1-\varepsilon_{1}\right) \beta \eta E_{\mathrm{S}}}{\delta_{\mathrm{E}} \delta_{V}}-1\right) V .
\end{aligned}
$$

It is notable that in $\Phi, E_{\mathrm{S}} \leq K$ and consequently we get,

$$
\frac{\mathrm{d} L}{\mathrm{~d} t}=\left(R_{0}-1\right) V
$$

The expression (2.10) reveals that the $\mathrm{d} L / \mathrm{d} t$ would be zero or negative only if $R_{0} \leq 1$. Let us define the subset $S$ of $\Phi$ where $\mathrm{d} L / \mathrm{d} t=0$ as $t \rightarrow \infty$ and is given by the equations

$$
\begin{aligned}
& V=0 \quad \text { for } \quad R_{0}<1, \\
& V=0, \quad \text { or } \quad E_{\mathrm{S}}=K \quad \text { for } \quad R_{0}=1
\end{aligned}
$$

It is observed that the maximum invariant set $\mathrm{Q}$ contained in the set $S$ is the plane $E_{\mathrm{I}}=0$ and $V=0$. Therefore $S=P_{0}(K, 0,0)$ and solutions started in $\mathrm{Q}$ approaches to $P_{0}(K, 0,0)$ as $t \rightarrow \infty$. According to Lyapunov-LaSalle theorem and LaSalle's Invariant 
Principle [32,33], the disease-free steady state is globally asymptotically stable and all solution trajectories started in $\Phi$ approaches to $P_{0}(K, 0,0)$ as $t \rightarrow \infty$. Hence the proof is completed. From the biological point of view, we can conclude that the infection can be removed if the basic reproduction is below its threshold value 1 .

\subsection{Sensitivity}

In this subsection, sensitivity analysis has been executed to elucidate the relative weightage and the robustness of predictions to the baseline parameters associated to the basic reproduction number $\left(R_{0}\right)$ [34]. Sensitivity analysis advantages to emphasize those parameters which are highly sensitive comparative to others in COVID-19 infection and we should pay attention to them to yield proper intervention schemes. The forward sensitivity indices of $R_{0}$ are given by

$$
\begin{aligned}
\Pi_{R_{0}}^{\beta} & =\frac{\partial R_{0}}{\partial \beta} \times \frac{\beta}{R_{0}}=\frac{\left(1-\varepsilon_{1}\right)\left(1-\varepsilon_{2}\right) \eta K}{\delta_{V}} \times \frac{\beta}{R_{0}}, \\
\Pi_{R_{0}}^{\eta} & =\frac{\partial R_{0}}{\partial \eta} \times \frac{\eta}{R_{0}}=\frac{\left(1-\varepsilon_{1}\right)\left(1-\varepsilon_{2}\right) \beta K}{\delta_{V}} \times \frac{\eta}{R_{0}}, \\
\Pi_{R_{0}}^{K} & =\frac{\partial R_{0}}{\partial K} \times \frac{K}{R_{0}}=\frac{\left(1-\varepsilon_{1}\right)\left(1-\varepsilon_{2}\right) \eta \beta}{\delta_{V}} \times \frac{K}{R_{0}}, \\
\Pi_{R_{0}}^{\varepsilon_{1}} & =\frac{\partial R_{0}}{\partial \varepsilon_{1}} \times \frac{\varepsilon_{1}}{R_{0}}=-\frac{\left(1-\varepsilon_{2}\right) \eta \beta K}{\delta_{V}} \times \frac{\varepsilon_{1}}{R_{0}}, \\
\Pi_{R_{0}}^{\varepsilon_{2}} & =\frac{\partial R_{0}}{\partial \varepsilon_{2}} \times \frac{\varepsilon_{2}}{R_{0}}=-\frac{\left(1-\varepsilon_{2}\right) \eta \beta K}{\delta_{V}} \times \frac{\varepsilon_{2}}{R_{0}}, \\
\Pi_{R_{0}}^{\delta_{V}} & =\frac{\partial R_{0}}{\partial \delta_{V}} \times \frac{\delta_{V}}{R_{0}}=-\frac{\left(1-\varepsilon_{1}\right)\left(1-\varepsilon_{2}\right) \eta \beta K}{\delta_{V}^{2}} \times \frac{\delta_{V}}{R_{0}} .
\end{aligned}
$$

The normalized sensitivity indices can be independent or dependent on the baseline parameters.

In the sensitivity analysis of $R_{0}$, the most sensitive parameters are carrying capacity of cells $(K)$, rate of infection $(\beta)$ and number of free virus produced $(\eta)$. Since sensitivity indices of these parameters are all equal to 1 , hence we can conclude that due to $10 \%$ increases (or decreases) of the value of carrying capacity of cells, infection rate or number of free virus, the basic reproduction number increases (or decreases) by $10 \%$. Other important parameter is virus removal rate $\left(\delta_{V}\right)$. The decrease (or increase) of virus removal rate by $10 \%$ increases (or decreases) the basic reproduction number by $6.67 \%$. Increase (or decrease) of the effectiveness of immunosuppressive drugs by $10 \%$ decreases (or increases) the basic reproduction number by $9.23 \%$. Similarly increasing (or decreasing) of antiviral drug effectiveness by $10 \%$ decreases (or increases) the basic reproduction number by $7.24 \%$. Hence immunosuppressive drugs play a pivotal role to control the disease progression in comparison with antiviral drug.

In the next section we construct a model considering optimal control strategies based on host SARS-CoV2/COVID-19 model.

\section{Model with time dependent control}

Optimal control technique is the potent mathematical tool which is mainly used in infectious disease modelling [35-37]. By help of this mathematical tool we can find out optimal drug dosage for which the disease can be controlled. Our main aim is to minimize the number infected cells and to maximize the number of uninfected cells [38]. Here we present two control variables.

(i) $u_{1}(t)$ denotes the antiviral drug strategy (Strategy I) which block infection.

(ii) $u_{2}(t)$ represents the drug strategies (Strategy II) which block the production of virus particles.

Our main aim is to maximize the level of uninfected cells. Also we need to retain cost of drug therapy at its minimum level. Here control function $u_{1}(t)$ and $u_{2}(t)$ are normalized between 0 and 1 . Here the control class is defined as:

$$
\begin{aligned}
\mathcal{M} & =\left\{\left(u_{1}(t), u_{2}(t)\right): 0 \leq u_{1}(t), u_{2}(t)\right. \\
& \leq 1, \text { is Lebesgue measurable }\} .
\end{aligned}
$$

Our main object is to minimize the objective functional:

$$
\begin{aligned}
\mathcal{J}\left(u_{1}(t), u_{2}(t)\right)= & \int_{0}^{T}\left[A_{1} E_{\mathrm{I}}-A_{2} E_{\mathrm{S}}\right. \\
& \left.+B_{1} u_{1}^{2}(t)+B_{2} u_{2}^{2}(t)\right] \mathrm{d} t
\end{aligned}
$$

subject to the state system

$$
\begin{aligned}
\frac{\mathrm{d} E_{\mathrm{S}}}{\mathrm{d} t} & =r E_{\mathrm{S}}\left[1-\frac{E_{\mathrm{S}}}{K}\right]-\left[1-u_{1}(t)\right] \beta E_{\mathrm{S}} V, \\
\frac{\mathrm{d} E_{\mathrm{I}}}{\mathrm{d} t} & =\left[1-u_{1}(t)\right] \beta E_{\mathrm{S}} V-\delta_{\mathrm{E}} E_{\mathrm{I}}, \\
\frac{d V}{\mathrm{~d} t} & =\left[1-u_{2}(t)\right] \eta \delta_{\mathrm{E}} E_{\mathrm{I}}-\delta_{V} V,
\end{aligned}
$$

with this positive initial condition:

$$
E_{\mathrm{S}}(0)=E_{S}^{0}, E_{\mathrm{I}}(0)=E_{I}^{0}, V(0)=V^{0} .
$$

All the coefficients $A_{1}, A_{2}, B_{1}$, and $B_{2}$ are nonnegative and they represent weights on the benefit of the cost related to the objective functional.

\subsection{Existence of an optimal control}

In this subsection, the existence of optimal control for the system (3.3) is analyzed. We use Pontryagin Maximum Principle [38] to characterize an optimal control, first we have to prove the existence of an optimal control $[35,39]$.

Theorem 5 There exists an optimal control vector $\left(u_{1}^{*}(t), u_{2}^{*}(t)\right) \in \mathcal{M}$ with corresponding state solutions $\left(E_{\mathrm{S}}^{*}, E_{\mathrm{I}}^{*}, V^{*}\right)$ that minimizes the objective functional $\mathcal{J}\left(u_{1}(t), u_{2}(t)\right)$ defined by (3.1) [35]. 
Table 2 Sensitivity indices of basic reproduction number $\left(R_{0}\right)$

\begin{tabular}{llr}
\hline Parameter & \multicolumn{1}{c}{ Description } & Sensitivity index \\
\hline$K$ & Carrying capacity of cell & 1.0000 \\
$\beta$ & Rate of infection/transmission & 1.0000 \\
$\eta$ & Number of free virus produced & 1.0000 \\
$\delta_{V}$ & Virus removal rate & -0.6667 \\
$\varepsilon_{1}$ & Efficacy of immunosuppressive drug & -0.9231 \\
$\varepsilon_{2}$ & Efficacy of antiviral drug & -0.7241 \\
\hline
\end{tabular}

Fig. 4 Normalized forward sensitivity indices of $R_{0}$ with respect to model parameters

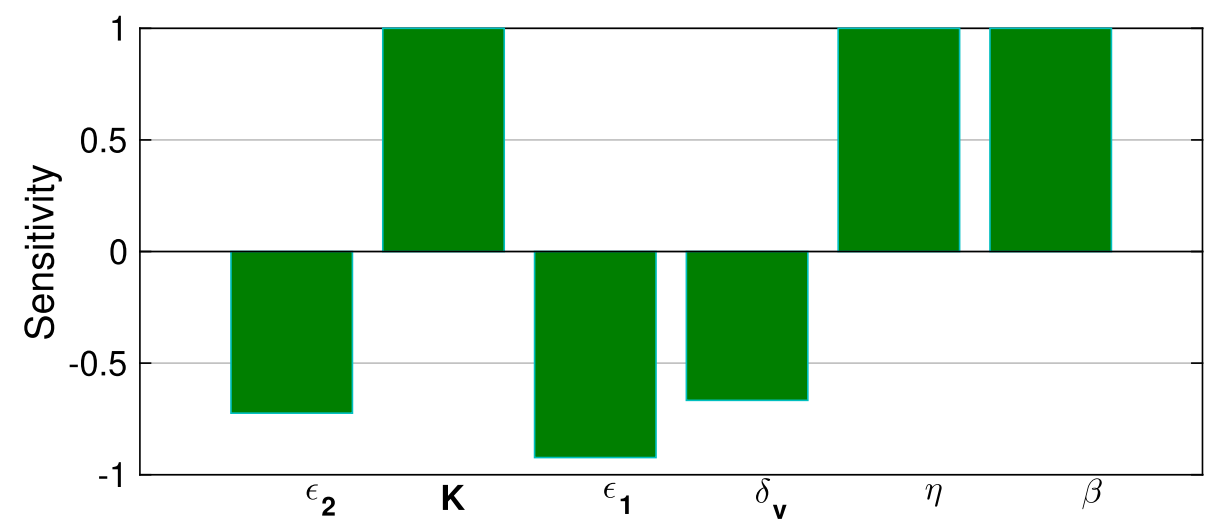

Proof All the solutions of the system (3.3) are nonnegative and uniformly bounded for given initial conditions (3.4). Non-negativity of objective functional values is obvious and non-negativity implies the boundedness of the objective functional. Hence a minimizing sequence of controls $\left(u_{1}^{v}(t), u_{2}^{v}(t)\right) \in \mathcal{M}$ exists such that;

$$
\lim _{v \rightarrow \infty} \mathcal{J}\left(u_{1}^{v}(t), u_{2}^{v}(t)\right)=\inf _{u_{1}(t), u_{2}(t) \in \mathcal{M}} \mathcal{J}\left(u_{1}(t), u_{2}(t)\right) .
$$

Therefore the controls belonging to $\mathcal{M}$ are uniformly bounded in $L^{\infty}$ and consequently they are uniform bounded in $L^{2}([0, T])$. Since the space $L^{2}([0, T])$ is reflexive [40], there exists $\left(u_{1}^{*}(t), u_{2}^{*}(t)\right) \in \mathcal{M}$ such that on a sub sequence,

$$
\begin{aligned}
& u_{1}^{v}(t) \rightarrow u_{1}^{*}(t), \quad u_{2}^{v}(t) \rightarrow u_{2}^{*}(t), \quad \text { weakly in } L^{2}([0, T]) \\
& \quad \text { as } v \rightarrow \infty
\end{aligned}
$$

Thus obviously the state sequence $\left(E_{\mathrm{S}}^{v}, E_{\mathrm{I}}^{v}, V^{v}\right)$ is uniformly bounded corresponding to $\left(u_{1}^{v}(t), u_{2}^{v}(t)\right)$. It can be seen that the uniform boundedness of the right-hand sides of the system (3.3) implies the uniform boundedness of the derivatives for $\left(E_{\mathrm{S}}^{v}, E_{\mathrm{I}}^{v}, V^{v}\right)$ and equicontinuity of the corresponding state sequence $\left(E_{\mathrm{S}}^{v}, E_{\mathrm{I}}^{v}, V^{v}\right)$. There exists $\left(E_{\mathrm{S}}^{*}, E_{\mathrm{I}}^{*}, V^{*}\right)$ (see ArzeliAscoli Theorem [35],) such that on a subsequence,

$\left(E_{\mathrm{S}}^{v}, E_{\mathrm{I}}^{v}, V^{v}\right) \rightarrow\left(E_{\mathrm{S}}^{*}, E_{\mathrm{I}}^{*}, V^{*}\right)$ uniformly on $[0, T]$.

Next, we choose the proper subsequence passing the limit to system (3.3) and corresponding to the controls $u_{1}^{*}(t), u_{2}^{*}(t)$, we can achieve the state solution
$\left(E_{\mathrm{S}}^{*}, E_{\mathrm{I}}^{*}, V^{*}\right)$. Then the lower semi-continuity of the $L^{2}$ - norm with respect to $L^{2}$ weak convergence implies that,

$$
\begin{aligned}
& \inf _{u_{1}(t), u_{2}(t) \in \mathcal{M}} \mathcal{J}\left(u_{1}(t), u_{2}(t)\right)=\lim _{v \rightarrow \infty} \mathcal{J}\left(u_{1}^{v}(t), u_{2}^{v}(t)\right) \\
& \geq \int_{0}^{T}\left(A_{1} E_{\mathrm{I}}-A_{2} E_{\mathrm{S}}+B_{1} u_{1}^{2}(t)+B_{2} u_{2}^{2}(t)\right) \\
& \mathrm{d} t=\mathcal{J}\left(E_{\mathrm{S}}^{*}, E_{\mathrm{I}}^{*}, V^{*}\right) .
\end{aligned}
$$

Therefore, $\left(E_{\mathrm{S}}^{*}, E_{\mathrm{I}}^{*}, V^{*}\right)$ is an optimal control.

Theorem 6 Define an optimal control vector $\left(u_{1}^{*}(t)\right.$, $\left.u_{2}^{*}(t)\right) \in \mathcal{M}$ and the corresponding state solutions $\left(E_{S}^{*}, E_{\mathrm{I}}^{*}, V^{*}\right)$ in the model (3.3), there exist adjoint variables $\vartheta_{1}(t), \vartheta_{2}(t)$, and $\vartheta_{3}(t)$, satisfying,

$$
\begin{aligned}
\vartheta_{1}^{\prime}=- & {\left[-A_{2}+\vartheta_{1} r\left(1-\frac{2 E_{\mathrm{S}}}{K}\right)+\left(\vartheta_{2}-\vartheta_{1}\right)\right.} \\
& \left.\left(1-u_{1}(t)\right) \beta V\right], \\
\vartheta_{2}^{\prime}=- & {\left[A_{1}-\vartheta_{2} \delta_{\mathrm{E}}+\vartheta_{3}\left(1-u_{2}(t)\right) \eta \delta_{\mathrm{E}}\right], } \\
\vartheta_{3}^{\prime}=- & {\left[\left(\vartheta_{2}-\vartheta_{1}\right)\left(1-u_{1}(t)\right) \beta E_{\mathrm{S}}-\vartheta_{3} \delta_{V}\right], }
\end{aligned}
$$

with the transversality conditions: $\vartheta_{1}(T)=0, \vartheta_{2}(T)$ $=0, \vartheta_{3}(T)=0$.

The optimal control vector $\left(u_{1}^{*}(t), u_{2}^{*}(t)\right)$ is given below

$$
\begin{aligned}
& u_{1}^{*}(t)=\min \left\{\max \left\{0, \frac{\left(\vartheta_{2}-\vartheta_{1}\right) \beta E_{\mathrm{S}} V}{2 B_{1}}\right\}, 1\right\}, \\
& u_{2}^{*}(t)=\min \left\{\max \left\{0, \frac{\vartheta_{3} \eta \delta_{\mathrm{E}} E_{\mathrm{I}}}{2 B_{2}}\right\}, 1\right\} .
\end{aligned}
$$


Proof To apply the Pontryagin's Minimum Principle, we consider the Hamiltonian as:

$$
\begin{aligned}
\mathcal{H}\left(E_{\mathrm{S}}, E_{\mathrm{I}}, V, \vartheta_{1}, \vartheta_{2}, \vartheta_{3}, u_{1}(t), u_{2}(t)\right) \\
=A_{1} E_{\mathrm{I}}-A_{2} E_{\mathrm{S}}+B_{1} u_{1}^{2}(t)+B_{2} u_{2}^{2}(t) \\
\quad+\vartheta_{1}\left[r E_{\mathrm{S}}\left(1-\frac{E_{\mathrm{S}}}{K}\right)-\left(1-u_{1}(t)\right) \beta E_{\mathrm{S}} V\right] \\
\quad+\vartheta_{2}\left[\left(1-u_{1}(t)\right) \beta E_{\mathrm{S}} V-\delta_{\mathrm{E}} E_{\mathrm{I}}\right] \\
\quad+\vartheta_{3}\left[\left(1-u_{2}(t)\right) \eta \delta_{\mathrm{E}} E_{\mathrm{I}}-\delta_{V} V\right]
\end{aligned}
$$

Now, adjoint variables $\vartheta_{1}(t)=0, \vartheta_{2}(t)=0, \vartheta_{3}(t)=$ 0 by:

$$
\vartheta_{1}^{\prime}=-\frac{\partial \mathcal{H}}{\partial E_{\mathrm{S}}}, \quad \vartheta_{2}^{\prime}=-\frac{\partial \mathcal{H}}{\partial E_{\mathrm{I}}}, \quad \vartheta_{3}^{\prime}=-\frac{\partial \mathcal{H}}{\partial V},
$$

with the transversality conditions $\vartheta_{1}(T)=0, \vartheta_{2}(T)=$ $0, \vartheta_{3}(T)=0$. We get the characterization of optimal controls by saying

$$
\frac{\partial \mathcal{H}}{\partial u_{1}}=0 \quad \text { and } \quad \frac{\partial \mathcal{H}}{\partial u_{2}}=0 .
$$

From $\partial \mathcal{H} / \partial u_{1}=0$ and $\partial \mathcal{H} / \partial u_{2}=0$, we get

$$
u_{1}(t)=\frac{\left(\vartheta_{2}-\vartheta_{1}\right) \beta E_{\mathrm{S}} V}{2 B_{1}}, \quad u_{2}(t)=\frac{\vartheta_{3} \eta \delta_{\mathrm{E}} E_{\mathrm{I}}}{2 B_{2}} .
$$

By considering the boundedness for $u_{1}(t)$ and $u_{2}(t)$ into account, the characterization of optimal controls are as follows:

$$
\begin{aligned}
& u_{1}^{*}(t)=\min \left\{\max \left\{0, \frac{\left(\vartheta_{2}-\vartheta_{1}\right) \beta E_{\mathrm{S}} V}{2 B_{1}}\right\}, 1\right\}, \\
& u_{2}^{*}(t)=\min \left\{\max \left\{0, \frac{\vartheta_{3} \eta \delta_{\mathrm{E}} E_{\mathrm{I}}}{2 B_{2}}\right\}, 1\right\} .
\end{aligned}
$$

Thus the proof is completed.

\section{Numerical simulation}

In this present section, we develop graphical displays of the fixed control system (2.2) and optimal control system (3.3), (3.4) and present the results. For fixed control problem, the drug efficacy $\varepsilon_{1}$ and $\varepsilon_{2}$ are updated. Also for time dependent control, the controls $u_{1}(t)$ and $u_{2}(t)$ are updated and are used to sole the state system and adjoint system together. Optimal control is a twopoint boundary value problem with separate boundary conditions at times $t=0$ and $t=T$. Here our aim is to solve this problem for the value of $T=100$. This is the value (in days) at which treatment is stopped. We use a finite difference approach to solve the optimality system (3.3)-(3.4) by applying the package BVP4C of MATLAB. To solve the problem easily we use $T=1$ and $t=0$. The solution of the problem for $T=1$ can be used as an initial guess to the solution nearby such that $T=1+\Delta T$, for $\Delta T$ sufficiently small. This process can be continued until the required problem is solved and this referred to as a homotopy path. By selecting different weight factors we can generate several treatment schedules for various time periods. Here we illustrate the 100 days treatment schedule. The optimal solution trajectories are displayed below in Figs. $(7,8,9)$ with different weight constants keeping all other parameters constant.

Here we study the following control strategies. To study the efficiency of the drug therapy, we consider three strategies as follows:

Strategy I: For this strategy, we observe that for fixed control (in Fig. 5) the total number of uninfected cells increases and infected cells number decreases with increasing value of the antiviral drug efficacy $\left(\varepsilon_{1}\right)$.

Figure 7 represents the effect of time dependent antiviral drug which blocks infection. In this figure, we observe that the number of infected cells along with virus level decrease as we keep the drug level at its maximum value throughout the treatment period.

Strategy II: For this strategy, we observe that for fixed control (in Fig. 6) the total number of uninfected cells increases and infected cells number decreases with increasing value of the antiviral drug efficacy $\left(\varepsilon_{2}\right)$.

Figure 8 represents the effect of time dependent antiviral drug which blocks new virus production. In this figure, we observe that the number of virus decreases as we keep the drug level at its maximum value throughout the treatment period. But in this strategy no such significant effect is observed in case of infected cells.

Strategy III: Figure 9 represents the effect of time dependent combination of antiviral drugs. In this figure, we observe that the number of infected cells along with virus level decreases as we keep the drug level at its maximum value throughout the treatment period. The outcome of this strategy is almost similar to the Strategy I.

\subsection{Efficiency analysis}

For Strategy I we consider $u_{1}(t) \neq 0, u_{2}(t)=0$, for Strategy II, $u_{1}(t)=0, u_{2}(t) \neq 0$ and for Strategy III we consider $u_{1}(t) \neq 0, u_{2}(t) \neq 0$. Here we shall calculate the efficiency index $\mathcal{E}$ which is defined as

$$
\mathcal{E}=\left(1-\frac{A^{C}}{A^{S}}\right) \times 100 \%
$$

where $A^{C}$ represents the area under the infected cells concentration as a function of time when the control is used and $A^{S}$ is the area under the infected total population curve in absence of control input. The cumulative number of infected cells during the time interval $[0,1]$ 
Fig. 5 The system

behaviour of Strategy I for fixed control

$\left(\varepsilon_{1} \neq 0, \varepsilon_{2}=0\right)$

Fig. 6 The system behaviour of Strategy II for fixed control $\left(\varepsilon_{1}=0, \varepsilon_{2} \neq 0\right)$

Fig. 7 Simulations of the SARS-CoV-2/COVID-19 model (3.3) showing the effect of the optimal strategies $\left(u_{1} \neq 0, u_{2}=0\right)$ for

$A_{1}=A_{2}=100, B_{1}=300$
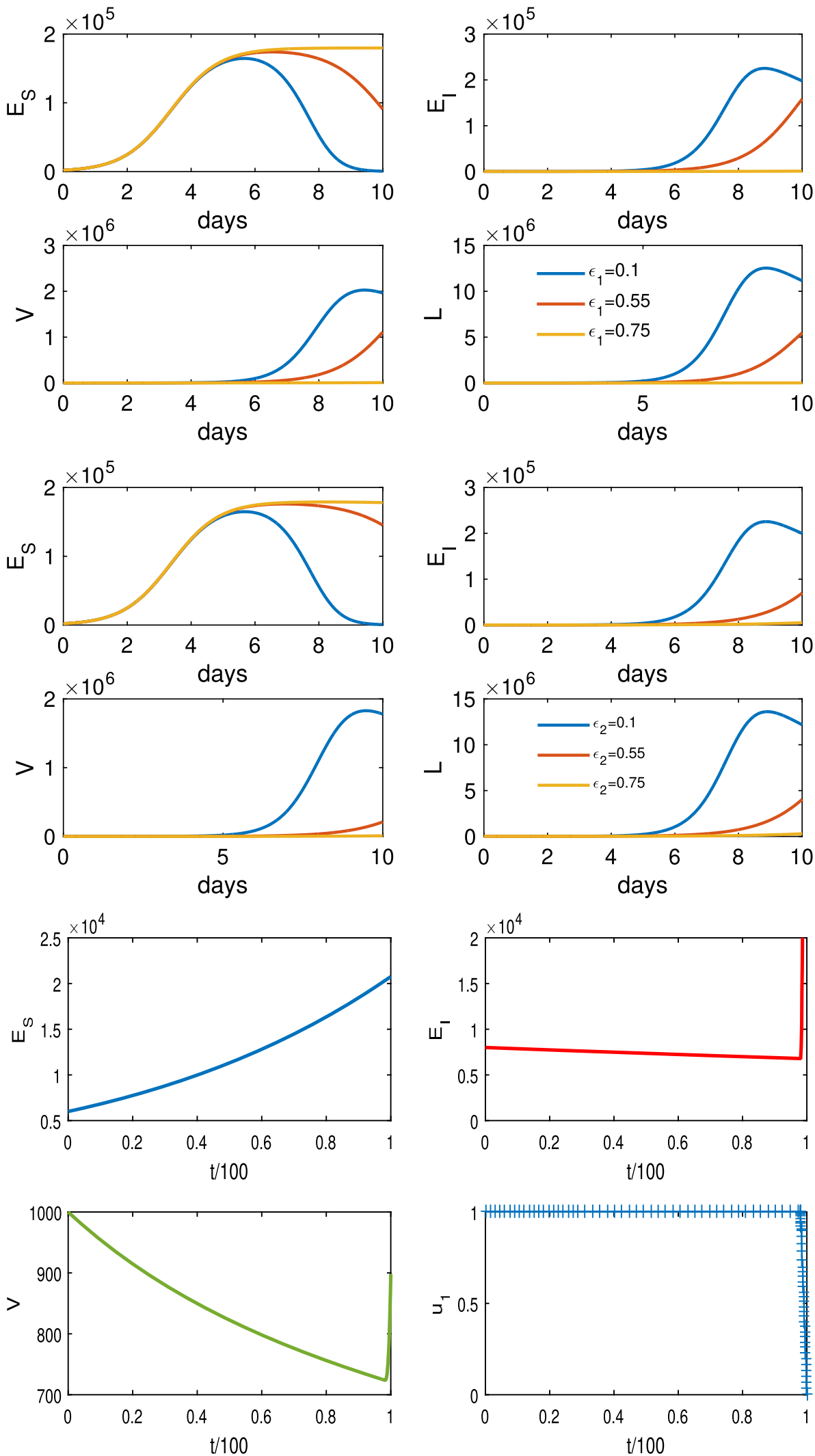
Fig. 8 Simulations of the SARS-CoV-2/COVID-19 model (3.3) showing the effect of the optimal strategies $\left(u_{1}=0, u_{2} \neq 0\right)$ for $A_{1}=A_{2}=100, B_{2}=$ 70000
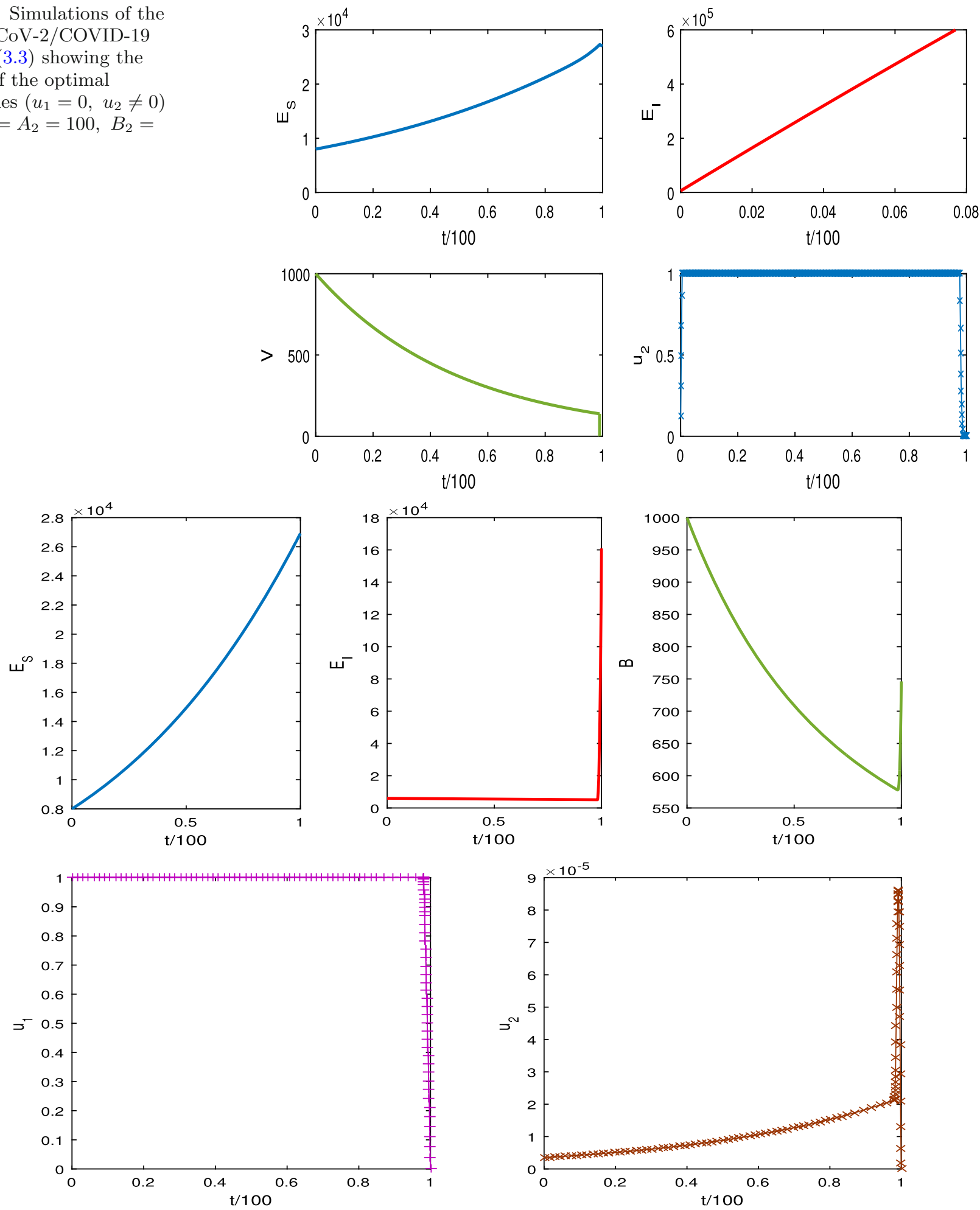

Fig. 9 Simulations of the SARS-CoV-2/ COVID-19 model (3.3) showing the effect of the optimal strategies $\left(u_{1} \neq 0, u_{2} \neq\right.$ 0 ) for $A_{1}=A_{2}=100, B_{1}=300, B_{2}=70000$ 
Table 3 Description of the different control strategies

\begin{tabular}{ll}
\hline Strategies & \multicolumn{1}{c}{ Description } \\
\hline I & Antiviral drug blocking infection \\
II & Antiviral drug blocking production of viral particles \\
III & Combination of antiviral drugs blocking infection and production \\
\hline
\end{tabular}

is defined by

$$
\mathbb{A}=\int_{0}^{1} E_{\mathrm{I}}(t) \mathrm{d} t
$$

By calculating the efficiency index we can adopt the best strategy whose efficiency index will be the biggest $[41,42]$. The values of $A^{C}$ and the efficiency indices for two strategies are given in Table (4).

From the Table (4), we can conclude that Strategy I is more effective than Strategy II. But Strategy III (which is the combination of two drugs) is the best strategy.

\subsection{Cost-effectiveness analysis}

To compare the differences between the costs and health outcomes of two alternative strategies, Incremental Cost-Effectiveness Ratio (ICER) plays a crucial role to select the best strategy. In the case of ICER, when we compare two competing strategies, the best strategy will be selected from less cost-effective ICER values. To quantify the cost-effectiveness, the Incremental Cost-Effectiveness Ratio (ICER) is defined as

$$
\text { ICER }=\frac{\text { The differences in intervention costs }}{\text { The differences in averted infection }} .
$$

Based on the control model simulation, we calculate the ICER for the strategies given below:

\begin{tabular}{llll}
\hline Strategies & Total infection averted & Total cost & ICER \\
\hline No control & 0 & 0 & - \\
I & 252.21 & 7231 & 28.67 \\
II & 317.74 & 6888 & -5.2342 \\
III & 798.90 & 1155 & -11.9189 \\
\hline
\end{tabular}

The ICER is calculated as follows:

$$
\begin{aligned}
\operatorname{ICER}(I) & =\frac{7231}{252.21}=28.6700, \\
\operatorname{ICER}(I I) & =\frac{6888-7231}{317.74-252.21}=-5.2342, \\
\operatorname{ICER}(I I I) & =\frac{1155-6888}{798.90-317.74}=-11.9189 .
\end{aligned}
$$

From the above Incremental Cost-Effectiveness Ratio (ICER) table, it is clearly observed that Strategy I is strongly dominated, which means that Strategy I is more costly and less effective than other strategies.
Therefore, Strategy I is excluded from the set of alternatives since it is consumed by limited resources. We recalculate ICER.

\begin{tabular}{llll}
\hline Strategies & Total infection averted & Total cost & ICER \\
\hline II & 317.74 & 6888 & 21.6800 \\
III & 798.90 & 1155 & -11.9149 \\
\hline
\end{tabular}

With this result, we can conclude that Strategy III (combination of drug therapy) has the least ICER and therefore it is more cost-effective than Strategy II. Hence the least cost-effective strategy is Strategy III. Also weight constants $B_{1}$ and $B_{2}$ play a pivotal role for choosing best strategy. Here, we analyse the strategies for a particular weight constant. Further analysis with different weight constants can give us more accurate results.

\section{Variable order fractional model of SARS-CoV-2 dynamic}

It has been proven that the fractions calculus is able to provide more accurate results compared to its integerorder counterpart. Hence, in this section, we propose a new variable-order fractional model that considers the SARS-CoV-2 dynamic. The proposed variable-order fractional model is given by

$$
\begin{aligned}
& { }_{0}^{\mathrm{c}} D_{t}^{q(t)} E_{\mathrm{S}}=r E_{\mathrm{S}}\left[1-\frac{E_{\mathrm{S}}}{K}\right]-\left[1-u_{1}(t)\right] \beta E_{\mathrm{S}} V, \\
& { }_{0}^{\mathrm{c}} D_{t}^{q(t)} E_{1}=\left[1-u_{1}(t)\right] \beta E_{\mathrm{S}} V-\delta_{\mathrm{E}} E_{\mathrm{I}}, \\
& { }_{0}^{\mathrm{c}} D_{t}^{q(t)} V=\left[1-u_{2}(t)\right] \eta \delta_{\mathrm{E}} E_{\mathrm{I}}-\delta_{V} V,
\end{aligned}
$$

where $q(t)$ denotes the time-varying fractional-order derivative. The fractional-order derivative is considered to change with respect to time as $q(t)=0.98+$ $0.01 \sin \frac{t}{5}$. Figure 10 shows the time history of the proposed variable order model. As it is shown in this figure, the value of fractional derivative significantly changes the behavior of the system.

\subsection{Optimal control}

In 1970s the genetic algorithm has been invented by John Holland [43]. To determine an optimum route, 
Table 4 Efficiency index for system (3.3)

\begin{tabular}{lll}
\hline Strategy & $\mathbb{A}=\int_{0}^{1} E_{\mathrm{I}}(t) \mathrm{d} t$ & $\sum=\left(1-\frac{A^{C}}{A^{S}}\right) \times 100 \%$ \\
\hline No control & $6.8125 \times 10^{5}$ & $0 \%$ \\
I & $8.3168 \times 10^{3}$ & $98.78 \%$ \\
II & $3.1575 \times 10^{6}$ & $53.65 \%$ \\
III & $6.4450 \times 10^{3}$ & $98.96 \%$ \\
\hline
\end{tabular}
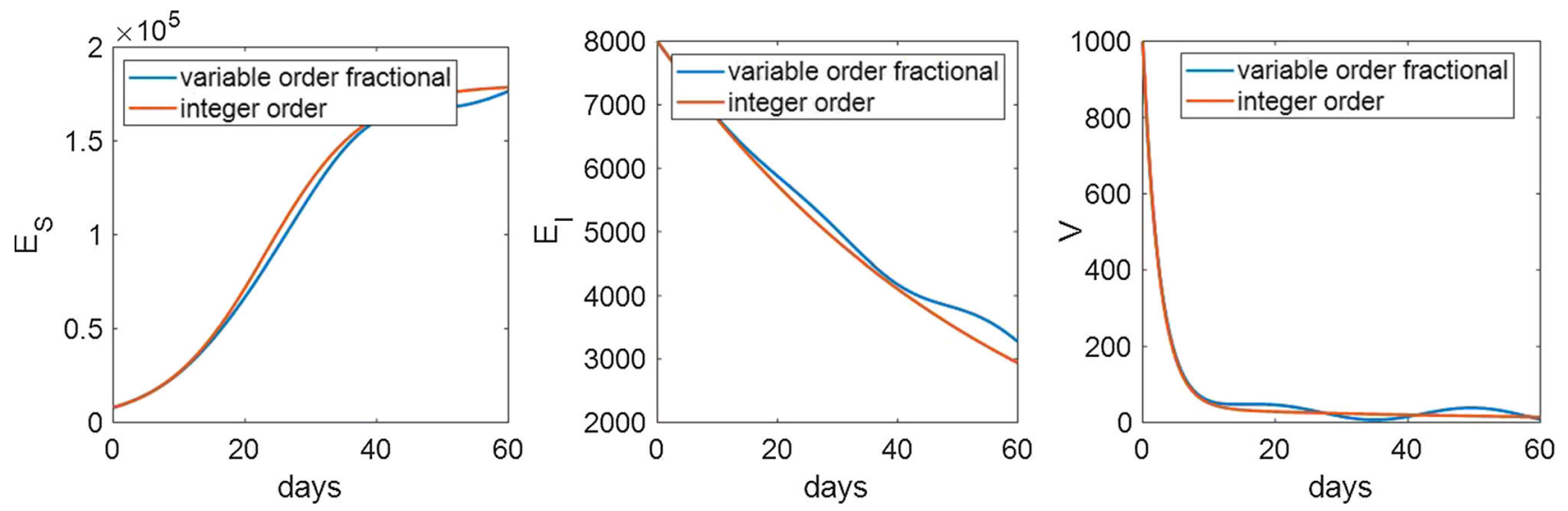

Fig. 10 The time history of the proposed model with different variable-order fractional models when $\varepsilon_{1}=0.2, \varepsilon_{2}=0$.

Table 5 Genetic algorithm configuration parameters

\begin{tabular}{ll}
\hline Parameter & Value \\
\hline Crossover fraction & 0.9 \\
Population size & 80 \\
Selection function & Tournament \\
Mutation function & Constraint-dependent \\
Crossover function & Intermediate \\
Migration direction & Forward \\
Migration fraction & 0.25 \\
Migration interval & 30 \\
Stopping criteria & 30,000 \\
\hline
\end{tabular}

the genetic algorithm follows an iterative and stochastic search approach. The genetic algorithm is established based on Darwin's survival-of-the-fittest method in evolution. This algorithm evolves superior solutions from a population of candidate solutions known as individuals. Based on a cost function, the population is assessed, and the best options for reproducing and mating to generate the next generation are chosen. To now, researchers have employed the genetic algorithm to handle a broad array of issues in many disciplines of study due to its interesting characteristics and strong convergence $[44,45]$. To identify optimum antiviral drug treatment, the genetic algorithm is applied. In this part, we use objective function (3.2). The results of the genetic algorithm are then used to obtain optimal antiviral drug treatment. The genetic algorithm's setup is shown in Table 5.

The objective function of the genetic algorithm is shown in Figure 11. As it is demonstrated, the applied algorithm successfully minimizes the objective function.

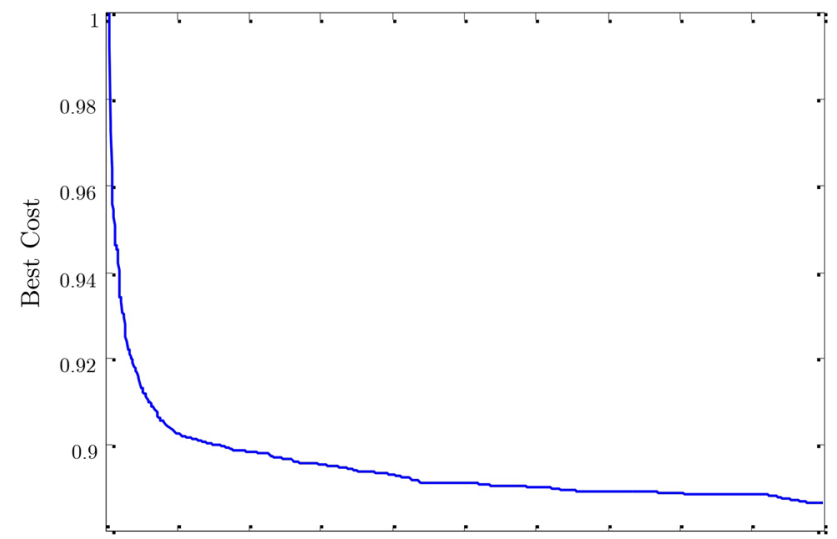

Fig. 11 The normalized value of best cost function

The optimal value for control inputs are obtained as $u_{1}=0.9989$ and $u_{2}=0.0045$.

The effect of the selected combination of the antiviral drug is demonstrated in Fig. 12. As shown in this figure, the number of infected cells along with viruses decreases throughout the treatment period. Also, through this optimal treatment, the production of viruses is blocked.

\section{Discussion}

We proposed the time-independent and time-dependent control problem to keep the susceptible cells level high and infected cells and virus level low. Also, our aim is to minimize drug costs. Here we use two control strategies. The first control input is described in terms of both sus- 

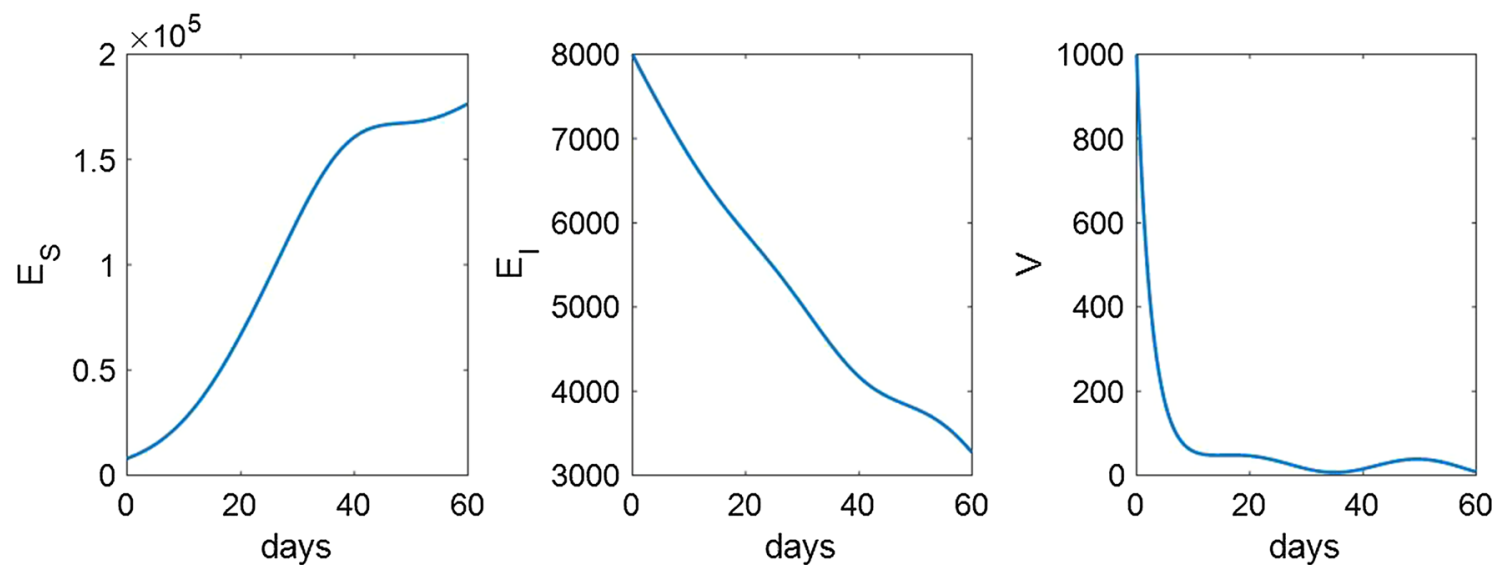

Fig. 12 Simulations of the variable order fractional SARS-CoV-2/COVID-19 model (5.1) showing the effect of the optimal strategies

ceptible cells and virus and their corresponding adjoint variables. It mainly blocks the infection or disease transmission. The second control input is described in terms of free virus generation and it acts to block new virus production.

We have derived the expression of the basic reproduction number by using the next-generation approach. By calculating the sensitivity index we have obtained that the most sensitive parameters are the carrying capacity of epithelial cells $(K)$, rate of infection $(\beta)$, and the number of free viruses produced $(\eta)$. PRCC results showed the negative correlation with the parameters $\delta_{V}, \varepsilon_{1}$, and $\varepsilon_{2}$. Thus if the drug efficacy or the removal rate of virus increases, the disease progression/ transmission reduces.

Here the existence condition for optimal control problem has been derived. We have also found the optimal treatment strategies by solving the corresponding optimal system. Through efficiency analysis, it is revealed that a combination of antiviral drug therapy is almost $95.24 \%$ effective compared to other strategies. Thus WHO suggests usage of the commonly used antiviral drugs to fight against the COVID-19 infection. As a result, the death rate would be restricted below $5 \%$ worldwide. Also, we have investigated the cost-effectiveness of the controls to determine the best strategies with minimum costs. By calculating ICER, we found that Strategy III i.e. combination of drugs therapy is the most effective therapy. Also, it has been observed that the Strategy I is effective in eliminating the disease. This strategy has the same effect as a combination of drug therapy. But this single drug strategy is not cost-effective as like as combination therapy.

\section{Conclusion}

In conclusion, SARS-CoV-2 infection poses significant threats to public health and we are struggling through it. In this paper, we have presented a deterministic model depicting the dynamics of SARS-CoV-2 and epithelial cells interaction. We have studied the model using optimal control theory holding an effective control measure against SARS-CoV-2 infection with the timedependent control mechanisms $\left(u_{1}(t), u_{2}(t)\right)$. Here $(1-$ $\left.u_{1}(t)\right)$ and $\left(1-u_{2}(t)\right)$ represent the efforts that prevent failure of the treatment in drug-sensitive SARS-CoV-2 infection. From our analytical and numerical findings the following results are observed:

(i). The model exhibits a disease-free equilibrium that is locally asymptotically stable if $R_{0}<1$ and would be unstable when $R_{0}>1$.

(ii). Carrying capacity $(K)$, rate of infection $(\beta)$, and number of new virions produced from the infected cells $(\eta)$ are the most sensitive parameters.

(iii). Antiviral drug therapy has a positive impact in reducing viral load. The total number of infected cells and virus load can be reduced by the application of time dependent control.

(iv). Both Strategy I and Strategy III are effective with $98 \%$ effective index.

$(v)$. Strategy III (combination of drug therapy) is the most cost-effective control strategy.

Finally, a variable fractional-order model of the COVID-19 Kinetics was introduced. In the proposed model, it is supposed that the value of the fractional derivative is changing with respect to time. Then, using the genetic algorithm optimization, an optimal treatment was proposed for the variable order fractional model. In a future study, we will investigate the control of the proposed variable order model through a multiobjective optimization method.

Acknowledgements This research was supported to Piu Samui by the Ph. D. research fellowship ("Swami Vivekananda Merit-cum-Means Scholarship") from the West Bengal Higher Education Department, Govt. of West Bengal, Bikash Bhavan, India with G.O. No. 52-Edn(B)/5B-15/2017 dated 07.06.2017.

Data Availability Statement All data used in this work have been obtained from official sources [46]. The data findings of this study are available from the corresponding author upon reasonable request. 


\section{References}

1. P. Zhou, X.-L. Yang, X.-G. Wang, B. Hu, L. Zhang, W. Zhang, H.-R. Si, Y. Zhu, B. Li, C.-L. Huang et al., A pneumonia outbreak associated with a new coronavirus of probable bat origin. Nature 579(7798), 270$273(2020)$

2. S.Q. Du, W. Yuan, Mathematical modeling of interaction between innate and adaptive immune responses in COVID-19 and implications for viral pathogenesis. J. Med. Virol. 92(9), 1615-1628 (2020)

3. A. Zeroual, F. Harrou, A. Dairi, Y. Sun, Deep learning methods for forecasting COVID-19 time-series data: a comparative study. Chaos Solitons Fractals 140,110121 (2020)

4. S.E. Eikenberry, M. Mancuso, E. Iboi, T. Phan, K. Eikenberry, Y. Kuang, E. Kostelich, A.B. Gumel, To mask or not to mask: modeling the potential for face mask use by the general public to curtail the COVID-19 pandemic. Infect. Dis. Model. 5, 293-308 (2020)

5. R.N. Thompson, Epidemiological models are important tools for guiding covid-19 interventions. BMC Med. 18, $1-4(2020)$

6. V.K.R. Chimmula, L. Zhang, Time series forecasting of COVID-19 transmission in Canada using LSTM networks. Chaos Solitons Fractals 135, 109864 (2020)

7. M.T. Meehan, D.P. Rojas, A.I. Adekunle, O.A. Adegboye, J.M. Caldwell, E. Turek, B.M. Williams, B.J. Marais, J.M. Trauer, E.S. McBryde, Modelling insights into the COVID-19 pandemic. Paediatr. Respir. Rev. 35, 64-69 (2020)

8. P. Samui, J. Mondal, S. Khajanchi, A mathematical model for COVID-19 transmission dynamics with a case study of India. Chaos Solitons Fractals 140, 110173 (2020)

9. J. Mondal, A.N. Chatterjee, Optimal control strategies of non-pharmaceutical and pharmaceutical interventions for covid-19 control, technical report (2020)

10. D. Aldila, M.Z. Ndii, B.M. Samiadji, Optimal control on covid-19 eradication program in Indonesia under the effect of community awareness. Math. Biosci. Eng 17(6), 6355-6389 (2020)

11. A. Yousefpour, H. Jahanshahi, S. Bekiros, Optimal policies for control of the novel coronavirus disease (covid19) outbreak. Chaos Solitons Fractals 136, 109883 (2020)

12. A. Beigi, A. Yousefpour, A. Yasami, J. Gómez-Aguilar, S. Bekiros, H. Jahanshahi, Application of reinforcement learning for effective vaccination strategies of coronavirus disease 2019 (covid-19). Eur. Phys. J. Plus 136(5), 1-22 (2021)

13. C. Tsay, F. Lejarza, M.A. Stadtherr, M. Baldea, Modeling, state estimation, and optimal control for the us covid-19 outbreak. Sci. Rep. 10(1), 1-12 (2020)

14. H. Jahanshahi et al., A new fractional-order hyperchaotic memristor oscillator: dynamic analysis, robust adaptive synchronization, and its application to voice encryption. Appl. Math. Comput. 383, 125310 (2020)

15. H. Jahanshahi, Smooth control of HIV/AIDS infection using a robust adaptive scheme with decoupled sliding mode supervision. Eur. Phys. J. Spec. Top. 227, 707718 (2018)
16. H. Jahanshahi et al., A new multi-stable fractional-order four-dimensional system with self-excited and hidden chaotic attractors: dynamic analysis and adaptive synchronization using a novel fuzzy adaptive sliding mode control method. Appl. Soft Comput. 87, 105943 (2020)

17. W.Z.R.A.S.B.H. Jahanshahi, A. Yousefpour, A financial hyperchaotic system with coexisting attractors: dynamic investigation, entropy analysis, control and synchronization. Chaos Solitons Fractals 126, 66-77 (2019)

18. H. Jahanshahi et al., On the development of variableorder fractional hyperchaotic economic system with a nonlinear model predictive controller. Chaos Solitons Fractals 144, 110698 (2021)

19. H. Jahanshahi, J.M. Munoz-Pacheco, S. Bekiros, N.D. Alotaibi, A fractional-order SIRD model with timedependent memory indexes for encompassing the multifractional characteristics of the COVID-19. Chaos Solitons Fractals 143, 110632 (2021)

20. H.M. Dobrovolny, Quantifying the effect of remdesivir in rhesus macaques infected with SARS-CoV-2. Virology 550, 61-69 (2020)

21. A.N. Chatterjee, F. Al Basir, A model for SARS-CoV-2 infection with treatment. Comput. Math. Methods Med. 2020, (2020)

22. A.N. Chatterjee, S. Saha, P.K. Roy, F. Al Basir, E. Khailov, E. Grigorieva, Insight of covid-19/SARSCOV-2 and its probable treatment - a mathematical approach (2020)

23. A.N. Chatterjee, B. Ahmad, A fractional-order differential equation model of covid-19 infection of epithelial cells. Chaos Solitons Fractals 147, 110952 (2021)

24. A.N. Chatterjee, F. Al-Basir, M.A. Almuqrin, J. Mondal, I. Khan, SARS-COV-2 infection with lytic and nonlytic immune responses: a fractional order optimal control theoretical study. Results Phys. 26, 104260 (2021)

25. C.A. Beauchemin, A. Handel, A review of mathematical models of influenza a infections within a host or cell culture: lessons learned and challenges ahead. BMC Public Health 11(S1), S7 (2011)

26. J. Nicholls, M. Chan, W. Chan, H. Wong, C. Cheung, D. Kwong, M. Wong, W. Chui, L. Poon, S. Tsao et al., Tropism of avian influenza a (h5n1) in the upper and lower respiratory tract. Nat. Med. 13(2), 147-149 (2007)

27. D. Van Riel, V.J. Munster, E. De Wit, G.F. Rimmelzwaan, R.A. Fouchier, A.D. Osterhaus, T. Kuiken, H5n1 virus attachment to lower respiratory tract. Science 312(5772), 399-399 (2006)

28. X. Xu, P. Chen, J. Wang, J. Feng, H. Zhou, X. Li, W. Zhong, P. Hao, Evolution of the novel coronavirus from the ongoing Wuhan outbreak and modeling of its spike protein for risk of human transmission. Sci. China Life Sci. 63(3), 457-460 (2020)

29. S. Banerjee, R. Keval, S. Gakkhar, Modeling the dynamics of hepatitis c virus with combined antiviral drug therapy: interferon and ribavirin. Math. Biosci. 245(2), 235-248 (2013)

30. O. Diekmann, J.A.P. Heesterbeek, J.A. Metz, On the definition and the computation of the basic reproduction ratio $\mathrm{r} 0$ in models for infectious diseases in heterogeneous populations. J. Math. Biol. 28(4), 365-382 (1990) 
31. P. Van den Driessche, J. Watmough, Reproduction numbers and sub-threshold endemic equilibria for compartmental models of disease transmission. Math. Biosci. 180(1-2), 29-48 (2002)

32. P. Srivastava, M. Banerjee, P. Chandra, Modeling the drug therapy for HIV infection. J. Biol. Syst. 17(02), 213-223 (2009)

33. P.K. Srivastava, P. Chandra, Modeling the dynamics of $\mathrm{HIV}$ and CD4+ t cells during primary infection. Nonlinear Anal. Real World Appl. 11(2), 612-618 (2010)

34. S. Chowdhury, P.K. Roy et al., Mathematical modelling of enfuvirtide and protease inhibitors as combination therapy for HIV. Int. J. Nonlinear Sci. Numer. Simul. 17(6), 259-275 (2016)

35. Q. Huang, X. Huo, S. Ruan, Optimal control of environmental cleaning and antibiotic prescription in an epidemiological model of methicillin-resistant Staphylococcus aureus infections in hospitals. Math. Biosci. 311, 13-30 (2019)

36. A.N. Chatterjee, P.K. Roy, Anti-viral drug treatment along with immune activator IL-2: a control-based mathematical approach for HIV infection. Int. J. Control 85(2), 220-237 (2012)

37. P.K. Roy, A.N. Chatterjee, Effect of HAART on CTL mediated immune cells: an optimal control theoretic approach. In Electrical Engineering and Applied Computing, pp. 595-607 (Springer, 2011)

38. L.S. Pontryagin, Mathematical Theory of Optimal Processes (Routledge, London, 2018)

39. M.R. Kelly Jr., J.H. Tien, M.C. Eisenberg, S. Lenhart, The impact of spatial arrangements on epidemic disease dynamics and intervention strategies. J. Biol. Dyn. 10(1), 222-249 (2016)
40. M. Schechter, Principles of Functional Analysis, no. 36. American Mathematical Society (2001)

41. S.A. Carvalho, S.O. da Silva, I. da Cunha Charret, Mathematical modeling of dengue epidemic: control methods and vaccination strategies. Theory Biosci. 138(2), 223-239 (2019)

42. H. Abboubakar, J.C. Kamgang, L.N. Nkamba, D. Tieudjo, Bifurcation thresholds and optimal control in transmission dynamics of arboviral diseases. J. Math. Biol. 76(1-2), 379-427 (2018)

43. J.H. Holland, The Dynamics of Searches Directed by Genetic Algorithms. Evolution, Learning and Cognition, pp. 111-127 (World Scientific, Singapore) (1988)

44. F.K.Q. Arakawa, K. Hasegawa, Study of anti-HIV HEPT analogues based on multi-objective genetic programming and counter-propagation neural network. In Chemometrics and Intelligent Laboratory System, pp. 91-98 (2006)

45. T.H. Wang, H.C. Wu, J.H. Meng, W.M. Yan, Optimization of a double-layered microchannel heat sink with semi-porous-ribs by multi-objective genetic algorithm. International Journal of Heat and Mass Transfer. 149, 119217 (2020)

46. R. Wolfel, V. Corman, W. Guggemos et al., Virological assessment of hospitalized cases of coronavirus disease. Nature 581, 465-9 (2020) 